\title{
Projet combiné ; stage de formation PE à la Cité des Sciences et classe « Villette »
}

Dominique Passelaigue et Brigitte Roussel

\section{(2) OpenEdition}

1 Journals

Édition électronique

URL : http://journals.openedition.org/trema/1493

DOI : 10.4000/trema.1493

ISSN : 2107-0997

Éditeur

Faculté d'Éducation de l'université de Montpellier

\section{Édition imprimée}

Date de publication : 1 janvier 2003

Pagination : 211-219

ISSN : 1167-315X

\section{Référence électronique}

Dominique Passelaigue et Brigitte Roussel, «Projet combiné ; stage de formation PE à la Cité des

Sciences et classe «Villette » », Tréma [En ligne], 20-21 | 2003, mis en ligne le 01 avril 2003, consulté le 08 mai 2019. URL : http://journals.openedition.org/trema/1493 ; DOI : 10.4000/trema.1493

Ce document a été généré automatiquement le 8 mai 2019.

Trema 


\title{
Projet combiné ; stage de formation PE à la Cité des Sciences et classe « Villette»
}

\author{
Dominique Passelaigue et Brigitte Roussel
}

\section{Formes usuelles des stages proposés par la Cité des Sciences et de l'Industrie}

1 La Cité des Sciences et de l'Industrie propose des formations, subventionnées en partie par la MAIF, aux professeurs des écoles en formation dans les IUFM, dont l'une d'entre elles, intitulée «de la Cité à l'école», axe ses objectifs sur le travail autour du musée interactif avec la classe.

2 Les stages standards habituellement proposés par la Cité des Sciences aux groupes de professeurs stagiaires sont gérés et programmés par le personnel de formation de La Villette, à savoir que les contenus de formation et les emplois du temps sont entièrement définis ; les types d'ateliers à préparer sont très cadrés et la mise en œuvre de ceux-ci est effectuée avec des classes d'enfants présentes sur le site (donc inconnues des stagiaires). Ce type de stage « clés en mains » est certes très séduisant étant donné que tout est prévu et demande très peu d'investissements aussi bien pour les formateurs que pour les stagiaires mais ne répond à aucune demande de projet de formation ciblée de la part de l'IUFM.

3 Un autre type de formation est proposée par La Villette, à savoir le stage " projet ». Dans cette proposition, il n'est en aucun cas question de travailler en synergie avec des enfants.

La première proposition ne nous convenait pas en regard de nos choix en terme de formations.

En effet, il est indispensable que les stagiaires décident les objectifs scientifiques à atteindre en fonction d'un projet scientifique élaboré dans une classe en partenariat avec l'enseignant titulaire. Un projet n'a de sens que s'il est fondé sur une interaction entre les 
acteurs du projet et une situation à laquelle ils se confrontent, qu'ils cherchent à modifier. Les groupes de professeurs stagiaires risquent de retrouver une situation de formation trop proche de celles qu'ils vivent à l'IUFM alors que l'autonomie développée à travers une implication importante dans le projet est l'un des objectifs principaux de cette action.

La mise en œuvre d'ateliers scientifiques avec des élèves que les professeurs stagiaires ne connaissent pas est en contradiction avec le travail de préparation de séquences habituellement effectué à l'IUFM. Cette situation ne permet pas de retour de la part des élèves, premiers intéressés par nos actes d'enseignements, et indicateurs du degré de nos réussites.

comme ce musée propose des classes de découverte sur projet ou à thème à des enseignants connaissant la cité et ayant fait un stage préparatoire, il semble possible de proposer un projet original défini par l'IUFM tenant compte à la fois des classes de découverte à l'attention d'enseignants en poste avec leurs élèves et des possibilités offertes par le stage projet, à destination des professeurs d'école stagiaires.

\section{Une façon différente de voir}

6 La nécessité pour la formation de "s'appuyer sur une articulation forte de la pratique de la classe et de l'éclairage théorique qui la fonde et qui en émerge " (Develay M., 1994, p. 76), de développer les liens IUFM - terrain et les propositions de La Villette ont soulevé les problèmes suivants ;

- Ne pourrait-on pas utiliser les ressources d'un espace muséologique dont les orientations sont pédagogiques pour mettre les PE dans des situations de professionnalisation à travers des réalisations pédagogiques et l'analyse des pratiques ce travail se faisant avec des enfants qu'ils connaissent et qu'ils suivent tout au long de leur année de formation?

- La classe de découverte ne pourrait-elle pas être organisée par les PE afin de compléter la formation sur le plan de l'organisation de ce type de séjours?

7 C'est cette option de travail en partenariat avec la Cité des Sciences que nous avons retenue pour la troisième année consécutive pour les raisons suivantes ;

- Le groupe de stagiaires est entièrement responsable des contenus scientifiques qu'ils travailleront toute l'année avec une classe nîmoise.

- Les besoins sont donc définis par les stagiaires, en fonction des enfants.

- Les demandes de " prestations » auprès du musée sont ciblées et sont en cohérence avec les axes définis par les stagiaires.

- L'équipe de formation de la Cité des Sciences doit alors répondre à une demande pédagogique authentique ce qui induit une relation qui relève plus du partenariat que de la prestation de services de formation entre la Cité des Sciences et l'IUFM.

8 Les deux groupes (classe de cycle 3 et professeurs stagiaires) travaillant en synergie en retireront des bénéfices mutuels ;

- La classe vit des projets de découvertes scientifiques sur l'année menés et encadrés par les PE et les enseignants de la classe, avec pour temps forts une semaine dans un lieu spécifique à Paris et communication par web aux parents et autres classes de l'école,

- Les PE2 travaillent sur l'organisation et la mise en place d'une classe transplantée, la participation tout au long de l'année à des projets de découvertes scientifiques avec les élèves, l'appropriation des ressources de la Cité, le montage d'animations spécifiques et de 
dispositifs pédagogiques, l'analyse des réalisations et la recherche de transferts possibles en classe.

9 Il faut noter que le stage de formation de l'IUFM de Nîmes est à ce jour le seul projet « indépendant » proposé à la Cité des Sciences!

\section{Les points forts du projet}

Le projet se déroule en trois étapes clés.

\subsection{Avant le séjour}

11 Il s'agit d'organiser matériellement la classe transplantée, à savoir, rechercher les moyens de transports les plus appropriés et en accord avec le budget des familles, un lieu d'hébergement adapté aux différents moments de la classe transplantée et agréé par l'institution, connaître les documents administratifs indispensables. Cela permet aux PE de prendre conscience de l'important travail à réaliser en amont de la mise en place des classes transplantées.

Répondre aux contraintes administratives, rencontrer les parents d'élèves, résoudre les problèmes du financement du séjour sont partie intégrante de la réalisation d'un tel projet. L'intérêt de travailler sur une situation réelle a confronté les PE avec les réalités du métier d'enseignant tant au niveau de l'organisation logistique que de la responsabilité pénale.

Parallèlement à ces différentes actions, les PE participent au lancement du projet scientifique avec les élèves et à la découverte des domaines proposés par la Cité (visite du site Internet, lecture des brochures de La Villette, choix des thèmes à étudier).

13 L'accent a été mis ici sur le fait qu'un centre de ressource tel un musée n'est pas autosuffisant dans la réussite d'une action. En effet, il n'est pas toujours évident pour les PE de comprendre qu'il est nécessaire d'effectuer une recherche documentaire très importante sur les possibilités offertes par un site muséologique. D'autre part, il ne faut pas oublier que les structures et espaces proposés par les sites sont souvent destinés à un public très large non spécifiquement d'origine scolaire.

14 Accompagnés par les différents formateurs, les PE ont en charge les prises de représentations auprès des élèves, la préparation des ateliers scientifiques et la mise en œuvre de ceux-ci dans la classe.

Cinq thèmes ont été choisis ; la lumière, l'astronomie, l'eau, l'électricité et les engrenages. Les questionnements au niveau des prises de représentations ont été élaborés par les PE ; il était important de mettre en application le fait qu'une séquence d'enseignement se construit à partir d'une réelle prise de représentation au niveau des élèves et que le rôle de l'enseignant est de préparer des ateliers susceptibles de répondre à des vraies questions pour les élèves.

"On ne peut enseigner une notion sans procéder à un état des lieux de ce qu'en pensent les élèves et à en tirer les conséquences " de plus «favoriser l'expression des représentations [...] contribue à les transformer» (Astolfi, Perterfalvi et Vérin, 1998, p. 73). C'est à travers la mise en évidence d'explications différentes de la sienne, à travers les conflits socio-cognitifs ainsi mis en place, que germera le problème scientifique à résoudre et le besoin de le résoudre. 

(sur plusieurs semaines) animés par un PE; chaque mise en œuvre était observée par d'autres PE, ce qui a permis d'effectuer des analyses critiques et aussi des remédiations successives étant donné que chaque atelier était effectué cinq fois. Cette évolution dans les prises de risques personnelles, les gestes professionnels ont permis une analyse assez fine des dysfonctionnements successifs et des possibilités de réajustement sur les contenus et les déroulements des ateliers.

Il faut préciser que ce projet ouvre sur un travail en classe concernant des disciplines autres que les sciences au sens large, mais que celui ci est pris en charge par le maître de la classe.

\subsection{Pendant le séjour}

Le séjour s'articule autour du travail en équipe sur la préparation de séances utilisant les ressources proposées par le musée, de la réalisation de chaque séance par un PE en présence d'observateurs PE et formateurs. Un bilan avec les enfants par un PE ne faisant pas partie de ce groupe de travail (afin de ne pas influencer les réponses des enfants) permet de vérifier l'impact immédiat de l'action menée.

18 d'approfondir leur réflexion didactique et pédagogique. C'est l'un des moments les plus riches en terme de formation.

Comme pour toute action de formation, la réalisation d'écrits permet de structurer les compétences travaillées et d'envisager le partage de ce vécu avec les autres stagiaires de la promotion. élèves dans et hors des moments de classe.

Ces différentes actions ont apporté in situ un grand sens des réalités pédagogiques à savoir le distinguotrès net entre les séances d'apprentissages projetées au niveau de la préparation et le déroulement effectif de celles-ci. Les remarques des observateurs et les bilans effectués avec les enfants ont souvent déstabilisé les stagiaires dans leurs certitudes d'aboutissement au niveau des apprentissages visés; les débats qui ont suivi ont permis une première mutualisation des pratiques engagées tant au niveau des contenus, des objectifs visés et des gestes professionnels en jeu.

L'essentiel est que les stagiaires puissent conduire des situations, les analyser et évaluer immédiatement, ce qui a été fait et enfin de pouvoir tenter un nouvel essai dans une situation comparable, la pluralité des observateurs permettant d'affiner l'analyse.

\subsection{Après le stage}

Le retour à Nîmes ne signifie pas encore la fin du projet « Villette »; un nécessaire travail de synthèse des acquis est à mener avec les élèves.

La réalisation d'une exposition interactive dans l'école a été le support de cette synthèse.

Ce projet est un moyen pédagogique pour réinvestir les connaissances scientifiques acquises par les élèves pendant l'année. Le but est de présenter des stands d'informations (vie de la classe transplantée et acquis des ateliers scientifiques) et de construire des dispositifs expérimentaux à destination des autres élèves de l'école et aux parents d'élèves en fin d'année. Chaque stand est animé par un élève de la classe.

Tréma, 20-21 | 2003 
Au niveau des adultes, il s'agit de finaliser l'écrit mémoire collectif par les PE en vue d'une diffusion à l'intérieur du site pour les autres PE (ou futurs PE stagiaires) qui seraient intéressés par ce type d'action.

Notre projet est en effet porté par les stagiaires informés de l'expérience par les promotions précédentes. Cet écrit de communication permet au stagiaire de prendre la mesure de leurs vécus et de l'évolution de leurs compétences.

\section{Analyse contrastée de l'expérience}

\subsection{Concernant les stagiaires}

21 Les PE ont un regard positif sur cette opération en terme d'apport personnel professionnalisant ; l'alternance des mises en œuvre et de l'analyse sur une journée, ainsi que le regard pluriel dans l'analyse (formateurs IUFM, IMF, PE, animateurs Villette) permettent une certaine prise de distance vis-à-vis des pratiques engagées.

L'observation du comportement d'une classe «lâchée » sans projet dans un musée a mis en évidence pour les PE que les élèves n'appréhendent que le côté ludique du lieu et ne s'engagent pas dans des activités d'apprentissage. Les PE ont été surpris de constater que les élèves ne lisent pas les panneaux d'information et ne s'intéressent pas aux origines des situations scientifiques observées. Les comportements se réduisent à de simples manipulations face à l'interactivité proposée. Il n'y a ni recherche de compréhension du phénomène ni même souvent d'observations.

Le bilan mettant en évidence d'éventuelles inadéquations entre les objectifs annoncés et les acquis des enfants apporte des outils pragmatiques au niveau de la nature des apprentissages des élèves ainsi que les origines possibles de ces divergences.

L'analyse des types de questionnements scientifiques produits spontanément par les PE et les réponses apportées par les élèves a mis en exergue l'impact du caractère ouvert ou fermé des questions de l'enseignant et des conséquences sur le déroulement des séances. Les difficultés des enfants à produire des écrits qui ne soient pas seulement une réponse aux attentes du maître ; le compte-rendu quotidien, ont été mises en évidence de même que la place du contrat didactique dans la profession.

Un travail métacognitif avec les enfants, rare dans les situations habituelles de classe a pu être mis en place, alors qu'il joue un rôle essentiel dans l'appropriation des savoirs. "l'enseignant doit aider l'élève à prendre conscience [...] de la manière dont il s'est approprié ce savoir » (Develay, 1994, p. 34).

Les PE ont pris conscience de la nécessaire responsabilisation permanente de l'enseignant en classe de découverte et de la multiplicité des tâches spécifiques non scolaires à assumer dans ce cadre.

\subsection{Pour les formateurs}

Ce projet a permis ;

- de repérer les comportements des PE relativement à la relation adulte - enfants, aux actes professionnels ;

- d'analyser l'effet feed-back permettant un réajustement de la pratique de formation ;

- de comprendre la nécessité d'un certain niveau d'exigence des formateurs sans lesquelles les productions des PE restent médiocres ; 
- de mener un travail métacognitif avec les PE;

- de mettre en évidence une nette évolution dans la qualité des séances proposées au cours de l'année de formation;

- de se prévenir d'un risque de dispersion dû à la taille et à la richesse des expositions de la Cité des Sciences; des journées riches mais trop longues et trop denses en raison de la brièveté du séjour.

- les vagues de stage, l'organisation d'un stage projet avec La Cité combiné avec une classe Villette sont des contraintes très difficiles à gérer ;

- l'impossibilité pour les stagiaires de s'approprier réellement (à savoir autrement que sur brochure) l'espace muséologique riche, et surtout les lieux concernant les sujets d'étude choisis avec les enfants ;

- le manque de communications inter services de la Cité des Sciences crée une surcharge de travail dans la réalisation des plannings parallèles classe - PE ; les différents partenaires n'ont pas l'habitude de devoir gérer un stage projet et une classe de découverte de façon simultanée. Il s'ensuit des accrochages relatifs aux disponibilités des lieux de travail, des accès aux expositions...

- le coût restant à la charge de chaque PE est de 150 Euros. La participation financière des PE a peut-être l'avantage de sélectionner les PE les plus motivés par le projet mais la somme dépensée est trop importante. Certains PE ont le sentiment qu'ils financent leur formation et ce point de vue mérite d'être étudié ;

- la nécessité de diminuer le coût de l'opération nous amène à faire le choix du transport routier, ce qui réduit le temps réel de mise en activité, sur la Cité.

Un problème semblable concerne le nombre important d'heures d'investissement des formateurs (IUFM et terrain) et des PE au niveau de la préparation et de la réalisation de l'action. Cette action a été engagée dans une volonté de différenciation de la formation IUFM (les parcours personnalisés) avec un quota d'heures institutionnelles très modeste par rapport aux réalités horaires.

\section{Conclusion}

En terme de mode de formation des enseignants, cette action peut se classer dans la catégorie «formation personnalisée ». En effet, si on reprend la définition proposée par M. Develay, il s'agit d'auto-formation assistée, avec des temps d'accompagnement; la tâche commune entre le formé et le formateur « accompagnant » est de déterminer ce qui pose problème, et comme dans une démarche d'investigation, de développer des hypothèses, d'envisager des solutions, d'imaginer des mises en œuvre, de se donner des moyens d'actions en utilisant toutes les ressources formatives à disposition.

Le stage à la Cité des Sciences permet de mettre en relation toutes les pratiques (métier d'élève, PE stagiaire, IMF, PE, PIUFM, Animateur Villette). Le regard pluriel lors de l'analyse de ces différentes pratiques (tant sur le plan de la didactique des disciplines qu'à travers l'aspect pédagogique) permet d'améliorer les compétences de chacun et l'évolution des performances des stagiaires est significative.

Nous pensons que cette expérience peut être développée avec d'autres partenaires (si tant est que des moyens financiers soient octroyés). Des expériences pourraient en effet concerner d'autres musées interactifs, s'appuyer sur des projets pluridisciplinaires... Se pose alors la question des dimensions complémentaires pour la formation que cette nouvelle action peut apporter. « Pourquoi ne faire acquérir aux professeurs stagiaires que 
des compétences dans la didactique et/ou la pédagogie d'une discipline ? Pourrions-nous souhaiter plus largement approfondir une approche transversale et interdisciplinaire du projet?»

Il nous semble nécessaire d'étudier plus finement les différents points de vue des chercheurs sur la professionnalisation du métier d'enseignant et l'analyse de pratique afin d'augmenter l'efficacité de ce type de projet et d'étendre sa mise en place.

En conclusion, nous pouvons affirmer que la prise en charge partielle dans la mise en place et la gestion d'une classe de découverte en partenariat avec un musée interactif est un support de formation riche et plébiscité par tous les partenaires. Même si cette situation induit des surcharges de travail et de stress, nous relançons chaque année cette opération. Nous pourrons ainsi affiner notre regard sur cette action et approfondir notre analyse théorique.

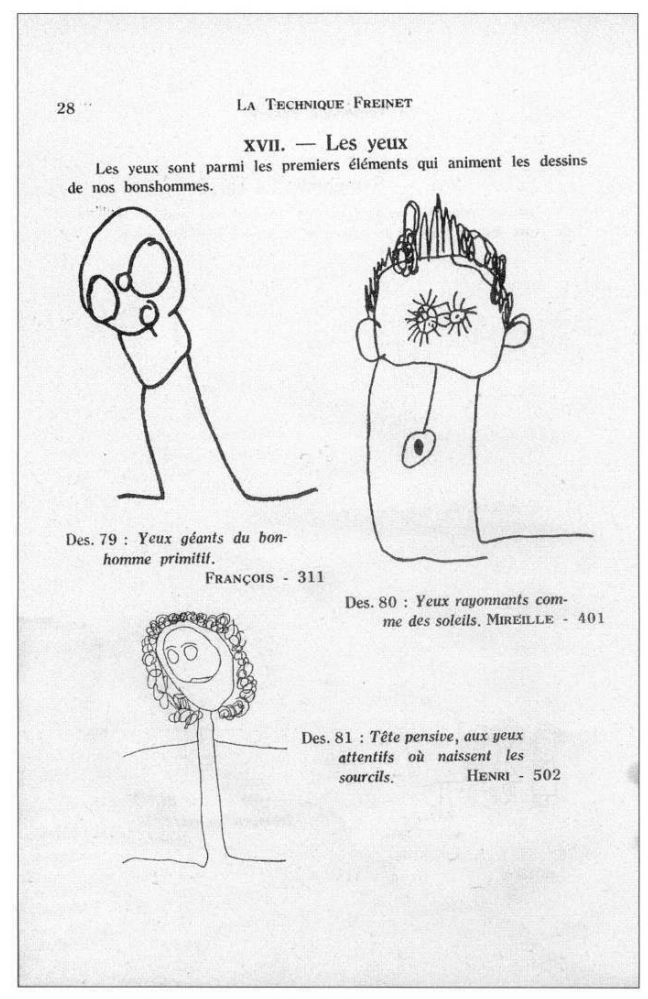

FREINET C. et al. ; LA gÉNÈse DE L'Homme. Cannes, Éditions de l'École Moderne Française (collection ; Brochures d'Éducation Nouvelle Populaire), № 79, Janvier 1953, p. 28.

\section{BIBLIOGRAPHIE}

DEVELAY M. ; « Peut-on former les enseignants ?», in Pédagogies. E.S.F, 1994.

ASTOLFI J.-P., PETERFALVI B. et VERIN A. ; Comment les enfants apprennent les sciences ?. Paris, Retz, 1998. 
PERRENOUD P. ; « Formation des maîtres et professionnalisation du métier », in Revue des sciences de l'éducation. Montréal (Canada), N 1, 1993.

\section{RÉSUMÉS}

Le projet a pour but de créer une action de formation visant la professionnalisation du métier d'enseignant en utilisant les ressources de la Cité des Sciences (La Villette - Paris). Il s'agit de mettre en relation étroite sur l'année un groupe de professeurs d'école stagiaires et une classe pour organiser un séjour scientifique et analyser les pratiques professionnelles.

The project's goal is to create a teacher training course having as its aim, improving the skills of the teaching profession and accomplishing this by making use of the resources of the "Cite des Sciences" museum at La Villette in Paris. The point is to bring together in a close relationship throughout the course of the school year, a group of student teachers and a class, in order to organize a course of science exploration field trips at the La Villette museum and to analyse the student teachers' teaching practices.

\section{INDEX}

Mots-clés : analyse de pratiques, partenariat, sciences

Keywords : analysis of teaching practices, partnership, sciences

\section{AUTEURS}

DOMINIQUE PASSELAIGUE

Professeur, IUFM de Montpellier

\section{BRIGITTE ROUSSEL}

Professeur, IUFM de Montpellier 